\title{
MORE ABOUT HOMOLOGICAL PROPERTIES OF PRECROSSED MODULES
}

\author{
NICK INASSARIDZE AND EMZAR KHMALADZE \\ (communicated by Graham Ellis)
}

\begin{abstract}
Homology groups modulo $q$ of a precrossed $P$-module in any dimensions are defined in terms of nonabelian derived functors, where $q$ is a nonnegative integer. The Hopf formula is proved for the second homology group modulo $q$ of a precrossed $P$-module which shows that for $q=0$ our definition is a natural extension of Conduché and Ellis' definition [CE]. Some other properties of homologies of precrossed $P$-modules are investigated.
\end{abstract}

\section{Introduction}

The homology of precrossed modules was introduced by Conduché and Ellis in [CE]. The aim of this paper is to pursue their line of investigation homological properties of precrossed modules.

Let $P$ be a group. A precrossed $P$-module $(M, \mu)$ is a group homomorphism $\mu: M \rightarrow P$ together with an action of $P$ on $M$ denoted by ${ }^{p} m$ for $p \in P$ and $m \in M$, which satisfies the following condition:

$$
\mu\left({ }^{p} m\right)=p \mu(m) p^{-1} .
$$

If in addition the following Peiffer identity holds

$$
{ }^{\mu(m)} m^{\prime}=m m^{\prime} m^{-1},
$$

$(M, \mu)$ is a crossed $P$-module (see e.x. [BH]).

A morphism $\varphi:(M, \mu) \rightarrow(N, \nu)$ of (pre)crossed $P$-modules is a commutative triangle

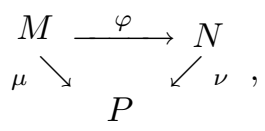

where $\varphi$ is a $P$-equivariant group homomorphism i.e. $\varphi\left({ }^{p} m\right)={ }^{p} \varphi(m)$ for all $m \in M, p \in P$. Let us denote the category of precrossed (crossed) $P$-modules by $\mathcal{P C M}(P)(\mathcal{C M}(P))$.

Further we shall occasionally supress explicit mention of the homomorphism $\mu$ in a precrossed $P$-module $(M, \mu)$ and write simply $M$.

Precrossed modules form a model of homotopy type in dimensions 1 and 2 for connected $\mathrm{CW}$-complexes. Precisly Kan's $\mathbb{G}$ functor establishes an equivalence relation between the category of connected $\mathrm{CW}$-complexes and the category of free simplicial groups $[\mathrm{K}]$ and the first two terms of the Moore chain complex associated to the simplicial group gives a precrossed module.

During this work both authors were supported by INTAS Georgia grant No 213 and NATO linkage grant No 975316. The first author was also supported by INTAS 93-436 and INTAS Fellowship grant for Young Scientists No 151.

Received 17 March 2000, revised 3 August 2000; published on 12 September 2000.

2000 Mathematics Subject Classification: 20J05, 18G10, 18G50

Key words and phrases: precrossed module, Peiffer abelianization, homology group, nonabelian derived functor (C) 2000, Nick Inassaridze and Emzar Khmaladze. Permission to copy for private use granted. 
Let $(M, \mu)$ be a precrossed $P$-module. The following type elements in $M$

$$
\left\langle m, m^{\prime}\right\rangle=m m^{\prime} m^{-1 \mu(m)} m^{\prime-1}, \quad m, m^{\prime} \in M
$$

are called Peiffer commutators, and now we give some identities for them from $[\mathrm{BC}]$

$$
\begin{array}{r}
\left\langle m, m^{\prime} m^{\prime \prime}\right\rangle=\left\langle m, m^{\prime}\right\rangle^{\mu(m)} m^{\prime}\left\langle m, m^{\prime \prime}\right\rangle^{\mu(m)} m^{\prime-1}, \\
\left\langle m m^{\prime}, m^{\prime \prime}\right\rangle=m\left\langle m^{\prime}, m^{\prime \prime}\right\rangle m^{-1}\left\langle m,{ }^{\mu\left(m^{\prime}\right)} m^{\prime \prime}\right\rangle, \\
{ }^{p}\left\langle m, m^{\prime}\right\rangle=\left\langle{ }^{p} m,{ }^{p} m^{\prime}\right\rangle, \\
\langle k, m\rangle=k m k^{-1} m^{-1}, \\
\langle k, m\rangle\langle m, k\rangle=k^{\mu(m)} k^{-1}
\end{array}
$$

for all $m, m^{\prime}, m^{\prime \prime} \in M, p \in P$ and $k \in K e r \mu$.

The Peiffer commutator subgroup $\langle M, M\rangle$, which is a subgroup of the group $M$ generated by the Peiffer commutators, plays the same role for precrossed modules as the commutator subgroup plays for groups. Analogously as a lower central series in a group, a lower Peiffer central series in a precrossed $P$-module is defined by Baues and Conduché $[\mathrm{BC}]$

$$
M^{(1)}=M \supset M^{(2)} \supset \ldots .
$$

This series has properties like classical central series giving a hope to generalize some methods of Curtis [CU1, CU2] and Quillen [Q] for nonsimply connected spaces.

The crossed $P$-module $\mu^{\prime}: M /\langle M, M\rangle \rightarrow P$ associated to the precrossed $P$-module $\mu$ : $M \rightarrow P$, where $M /\langle M, M\rangle$ is a factor group of $M$ by the Peiffer commutator subgroup, the homomorphism $\mu^{\prime}$ and the action of $P$ on $M /\langle M, M\rangle$ are induced by $\mu$ and the action of $P$ on $M$ respectively, plays the role of abelianization of a group which we further call Peiffer abelianization. As an analog of the classical first group homology, Conduché and Ellis [CE] defined the first homology of a precrossed $P$-module $(M, \mu)$ by Peiffer abelianization i.e.

$$
H_{1}(M)_{P}=M /\langle M, M\rangle .
$$

We point out that despite its name, the Peiffer abelianization can be nonabelian.

Let $(L, \lambda),(M, \mu)$ and $(N, \nu)$ be precrossed $P$-modules. A short exact sequence of groups

$$
1 \longrightarrow L \stackrel{\varphi}{\longrightarrow} M \stackrel{\psi}{\longrightarrow} N \longrightarrow 1
$$

is called short exact sequence of precrossed $P$-modules if $\varphi$ and $\psi$ are morphisms of the category $\mathcal{P C M}(P)$.

Let $X$ be a set and $\delta: X \rightarrow P$ a map to the group $P$. Then the free precrossed $P$-module $\partial: F \rightarrow P$ with base $(X, \delta)$ is defined as follows: $F$ is the free group generated by the set $X \times P, \partial$ is defined on generators by $\partial(x, p)=p \delta(x) p^{-1}$ and the action of $P$ on $F$ is given by $p\left(x, p^{\prime}\right)=\left(x, p p^{\prime}\right)$.

Conduché and Ellis in $[\mathrm{CE}]$ also defined the second homology group of a precrossed $P$ module $(M, \mu)$ by the Hopf formula

$$
H_{2}(M)_{P}=R \cap\langle F, F\rangle /\langle\langle F, R\rangle\rangle,
$$

where $1 \rightarrow R \rightarrow F \rightarrow M \rightarrow 1$ is a short exact sequence of precrossed $P$-modules, $(F, \partial)$ is a free precrossed $P$-module with some base $(X, \delta)$ which is called free presentation of the precrossed $P$-module $(M, \mu)$. They studied some properties so defined low dimensional homology groups of precrossed $P$-modules and hoped that higher homology could be defined analogously using Hopf formulas for higher homology groups (see $[\mathrm{BE}])$. Using this way to define all homology groups of a precrossed $P$-module $(M, \mu), H_{n}(M)_{P}$, one should have some difficulties, for $n \geqslant 3$, to prove that the definition does not depend on the free presentation of the precrossed $P$-module $(M, \mu)$.

In the present paper we have another conception to define all homology groups of a precrossed $P$-module, particulary the use of nonabelian derived functors. 
All treatments with homology of precrossed $P$-modules we consider in the $q$ modular aspect, where $q$ is a nonnegative integer, and for $q=0$ it gives homology groups of precrossed modules introduced in $[\mathrm{CE}]$. Thus, for nonnegative integer $q$, we define homology groups modulo $q$ of precrossed $P$-module $(M, \mu)$ in any dimension $n \geqslant 1$, denoted by $H_{n}(M, q)_{P}$, and study their properties generalizing the classical homology of groups with coefficients in $\mathbb{Z}_{q}=\mathbb{Z} / q \mathbb{Z}$.

\section{Construction}

Let us denote by $\operatorname{Set}(P)$ the category of sets over the group $P$, whose objects are all sets with a map to $\mathrm{P}$ and morphisms are all maps of sets such that the corresponding triangles are commutative.

Consider the functor $\mathcal{F}: \operatorname{Set}(P) \rightarrow \mathcal{P C M}(P)$ defined as follows: for an object $X \stackrel{\alpha}{\longrightarrow} P$ of the category $\operatorname{Set}(P)$, let $\mathcal{F}(X \stackrel{\alpha}{\longrightarrow} P)$ be a free precrossed $P$-module with base $(X, \alpha)$; for a morphism $X \stackrel{\kappa}{\longrightarrow} X^{\prime}$, let $\mathcal{F}(\kappa)$ be the canonical homomorphism induced by $\kappa$.

It is known that the forgetful functor from the category $\mathcal{P C M}(P)$ to the category $\operatorname{Set}(P)$ is a right adjoint of the functor $\mathcal{F}$. This adjunction induces the cotriple $(\mathcal{F}, \tau, \delta)$ in the category $\mathcal{P C M}(P)$. Let $\mathcal{P}$ be the projective class in the category $\mathcal{P C M}(P)$ induced by the cotriple $(\mathcal{F}, \tau, \delta)$ (see $[\mathrm{TV}],[\mathrm{IH}])$.

First we describe the projective class $\mathcal{P}$ and the corresponding $\mathcal{P}$-epimorphisms.

Proposition 1.1. A morphism $M \stackrel{\varphi}{\longrightarrow} N$ of the category $\mathcal{P C M}(P)$ is a $\mathcal{P}$-epimorphism if and only if $\varphi$ is surjective (as map of sets).

Proposition 1.2. In the category $\mathcal{P C M}(P)$ the following conditions are equivalent:

(i) A precrossed $P$-module $(Q, \nu)$ belongs to the projective class $\mathcal{P}$;

(ii) $(Q, \nu)$ is a free precrossed $P$-module with base $(X, \alpha)$ for some object $X \stackrel{\alpha}{\longrightarrow} P$ of the category $\operatorname{Set}(P)$

The proof of these propositions is left to the reader.

A precrossed $P$-module $(N, \nu)$ is a precrossed $P$-submodule of a precrossed $P$-module $(M, \mu)$ if $N$ is a subgroup of $M$, the action of $P$ on $N$ is induced by the action of $P$ on $M$ and $\nu$ is the restriction of $\mu$ on $N$. If, in addition, $N$ is a normal subgroup of the group $M$ then we write $N<_{P} M$.

Let $(M, \mu)$ be a precrossed $P$-module, $N, N^{\prime}$ be two subgroups of $M$ and $q$ be a nonnegative integer. We denote by $\left\langle N, N^{\prime}\right\rangle_{(q)}$ the subgroup of $M$ generated by the elements $\left\langle n, n^{\prime}\right\rangle$ and $k^{q}$ for all $n \in N, n^{\prime} \in N^{\prime}, k \in N \cap N^{\prime} \cap \operatorname{Ker} \mu$. Let $\left\langle\left\langle N, N^{\prime}\right\rangle\right\rangle_{(q)}=\left\langle N, N^{\prime}\right\rangle_{(q)}\left\langle N^{\prime}, N\right\rangle_{(q)}$. One has the following

Lemma 1.3. (i) If $N$ and $N^{\prime}$ are precrossed P-submodules of $M$ then $\left\langle N, N^{\prime}\right\rangle_{(q)}$ and $\left\langle\left\langle N, N^{\prime}\right\rangle\right\rangle_{(q)}$ are precrossed $P$-submodules of $M$.

(ii) If $N<_{P} M$ then $\langle M, N\rangle_{(q)}<_{P} M,\langle N, M\rangle_{(q)}<_{P} M,\langle\langle M, N\rangle\rangle_{(q)}<_{P} M$.

Proof. (i) Follows from the relation (3) and the equality ${ }^{p}\left(k^{q}\right)=\left({ }^{p} k\right)^{q}, p \in P, k \in N \cap N^{\prime} \cap$ $\operatorname{Ker} \mu$.

(ii) Follows from the relations (1), (2) and the equality $m k^{q} m^{-1}=\left(m k m^{-1}\right)^{q}, m \in M$, $k \in N \cap \operatorname{Ker} \mu$.

Using Lemma 1.3 one can define a covariant functor $T_{(q)}$ from the category $\mathcal{P C M}(P)$ to the category $\mathfrak{G r}$ of groups by the following way: for any precrossed $P$-module $(M, \mu)$, let $T_{(q)}(M)=M /\langle\langle M, M\rangle\rangle_{(q)}=M /\langle M, M\rangle_{(q)}$; for a morphism $(M, \mu) \stackrel{\varphi}{\longrightarrow}\left(M^{\prime}, \mu^{\prime}\right)$, let $T_{(q)}(\varphi)$ be a group homomorphism induced by $\varphi$. Note that for $q=0$ the functor $T_{(q)}$ is the Peiffer abelianization functor. 
In the category $\mathcal{P C M}(P)$ there exist finite limits (easy to show). Let us consider the nonabelian left derived functors $\mathcal{L}_{n}^{\mathcal{P}} T_{(q)}, n \geqslant 0$, of the functor $T_{(q)}: \mathcal{P C M}(P) \rightarrow \mathfrak{G r}$ relative to the projective class $\mathcal{P}$ induced by the cotriple $(\mathcal{F}, \tau, \delta)$ in the category $\mathcal{P C M}(P)[\mathrm{IH}]$.

Definition 1.4. Let $P$ be a group, $(M, \mu)$ be a precrossed $P$-module and $q$ be a nonnegative integer. Define the $n$-th homology group modulo $q$ of the precrossed $P$-module $(M, \mu)$ by

$$
H_{n}(M, q)_{P}=\mathcal{L}_{n-1}^{\mathcal{P}} T_{(q)}(M), \quad n \geqslant 1 .
$$

Proposition 1.5. Let $\mu: M \rightarrow P$ be a precrossed $P$-module such that $\mu(m)=1$ for all $m \in M$. Then one has

$$
H_{n}(M, q)_{P}=H_{n}\left(M, \mathbb{Z}_{q}\right), \quad n \geqslant 1 .
$$

Proof. Consider a $\mathcal{P}$-projective pseudosimplicial resolution (see $[\mathrm{IH}])$ of $(M, \mu)$ in the category $\mathcal{P C M}(\mathcal{P})$

$$
\cdots \underset{\vdots}{\longrightarrow} F_{2} \stackrel{\kappa_{2}}{\longrightarrow} Y_{1} \rightleftarrows F_{1} \stackrel{\kappa_{1}}{\longrightarrow} Y_{0} \longrightarrow F_{0} \rightarrow M
$$

where $F_{n} \in \mathcal{P}$ and $Y_{n}$ is a simplicial kernel in the category $\mathcal{P C M}(P)$. By Propositions 1 and 2 all $F_{n}$ are free groups and all $\kappa_{n}$ are surjective group homomorphisms, implying that (6) is a projective resolution of the group $M$ in the category $\mathfrak{G r}$. Since $\mu$ is a trivial group homomorphism, $T_{(q)}\left(F_{n}\right)=F_{n}^{a b} / q F_{n}^{a b}$. Using $[\mathrm{BB}]$ one gets the assertion.

\section{Main Results}

In this section we give our main results. We investigate the functor $T_{(q)}$ and prove a Hopf type formula for the second homology modulo $q$ of precrossed $P$-modules, generalizing the classical one (see $[\mathrm{BR}],[\mathrm{E}]$ ).

Let $\mathcal{C}$ be a category with finite limits, $\mathcal{Q}$ be a projective class in the category $\mathcal{C}$ and $T$ be a covariant functor from the category $\mathcal{C}$ to the category $\mathfrak{G} \mathfrak{r}$ of groups.

Definition $2.1([\mathbf{P}])$. The functor $T$ is called a cosheaf over $(\mathcal{C}, \mathcal{Q})$ if for any $\mathcal{Q}$-epimorphism $X \rightarrow A$ the sequence of groups

$$
T\left(X \times_{A} X\right) \longrightarrow T(X) \longrightarrow T(A) \longrightarrow 1
$$

is simplicially exact, where $X \times_{A} X$ is the pullback of the diagram

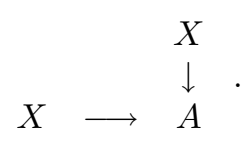

Lemma 2.2. Let $P$ be a group and $q$ be a nonegative integer. Then the functor $T_{(q)}: \mathcal{P C M}(P) \rightarrow \mathfrak{G r}$ is a cosheaf over $(\mathcal{P C M}(P), \mathcal{P})$, where $\mathcal{P}$ is the projective class induced by the cotriple $(\mathcal{F}, \tau, \delta)$ (see above).

Proof. It is easy to verify that for a short exact sequence of precrossed $P$-modules

$$
1 \longrightarrow L \longrightarrow M \longrightarrow N \longrightarrow 1
$$

there is an exact sequence of groups

$$
T_{(q)}(L) \longrightarrow T_{(q)}(M) \longrightarrow T_{(q)}(N) \longrightarrow 1
$$

Consider a $\mathcal{P}$-epimorphism $Q \stackrel{\alpha}{\longrightarrow} M$ in the category $\mathcal{P C M}(P)$. We have to show that the diagram of groups

$$
T_{(q)}\left(Q \times_{M} Q\right) \stackrel{d_{0}}{\underset{d_{1}}{\rightleftarrows}} T_{(q)}(Q) \stackrel{T_{(q)}(\alpha)}{\longrightarrow} T_{(q)}(M) \longrightarrow 1
$$


is exact. In effect, there is the following commutative diagram of groups

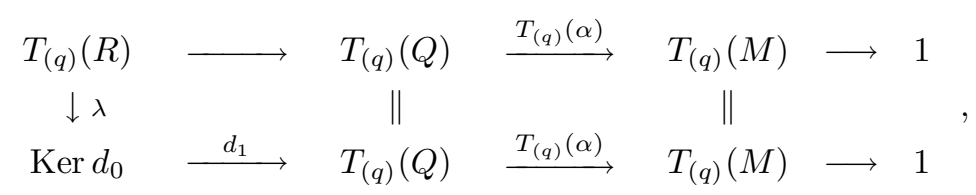

where $R$ is the kernel of $\alpha: Q \rightarrow M, \lambda$ is a homomorphism induced by the inclusion $R \hookrightarrow Q \times_{M} Q, r \mapsto(r, 1)$, and the top row is exact by (7). Hence the bottom row of this diagram is also exact.

Proposition 2.3. Let $P$ be a group, $(M, \mu)$ be a precrossed $P$-module and $q$ be a nonnegative integer. Then there is a natural isomorphism

$$
H_{1}(M, q)_{P} \approx M /\langle M, M\rangle_{(q)}
$$

Proof. Follows by Lemma 2.2 and [P or IH, Proposition 2.26].

Theorem 2.4 (Hopf Formula). Let $P$ be a group, $(M, \mu)$ be a precrossed $P$-module and $q$ be a nonnegative integer. Then there is an isomorphism

$$
H_{2}(M, q)_{P} \approx R \cap\langle F, F\rangle_{(q)} /\langle\langle F, R\rangle\rangle_{(q)},
$$

where $1 \longrightarrow R \longrightarrow F \stackrel{\varphi}{\longrightarrow} M \longrightarrow 1$ is any free presentation of the precrossed $P$-module $(M, \mu)$ i.e. using Propositions 1 and $2, F$ is an object of the projective class $\mathcal{P}$ and $\varphi$ is a $\mathcal{P}$-epimorphism.

Proof. Consider the Ĉech resolution of $(M, \mu) \in \mathcal{P C M}(P)$ for $\varphi: F \rightarrow M[\mathrm{P}$ or IH, Definition 2.31, Examples]

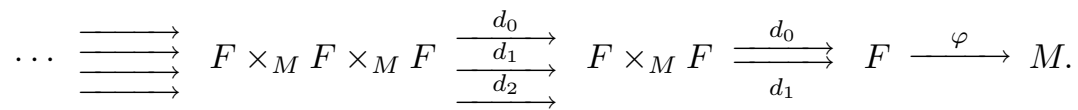

By Lemma $2.2 T_{(q)}$ is a cosheaf over $(\mathcal{P C M}(P), \mathcal{P})$ and using [P or IH, Theorem 2.39(ii)] there is an isomorphism

$$
\mathcal{L}_{1}^{\mathcal{P}} T_{(q)}(M) \approx \pi_{1} C_{*},
$$

where $C_{*}$ is the following simplicial group

$$
C_{*} \equiv \cdots \rightleftarrows T_{(q)}\left(F \times_{M} F \times_{M} F\right) \frac{\frac{T_{q}\left(d_{0}\right)}{T_{q}\left(d_{1}\right)}}{\stackrel{T_{q}\left(d_{2}\right)}{\longrightarrow}} T_{(q)}\left(F \times_{M} F\right) \underset{T_{q}\left(d_{1}\right)}{\stackrel{T_{q}\left(d_{0}\right)}{\rightleftarrows}} T_{(q)}(F)
$$

The Moore complex $N C_{*}$ of the simplicial group $C_{*}$ has length 1 i.e. $\left(N C_{*}\right)_{n}=0, n \geqslant 2$. This follows from the fact that the Moore complex of the Cech resolution has length 1.

Hence $\pi_{1} C_{*}=\operatorname{Ker} T_{q}\left(d_{0}\right) \cap \operatorname{Ker} T_{q}\left(d_{1}\right)$.

Furthermore, one has the following isomorphism of precrossed $P$-modules $F \times_{M} F \stackrel{\approx}{\longrightarrow} R \rtimes F$, defined by $(r, f) \mapsto(r f, f)$, where the precrossed $P$-module structure on the group $R \rtimes F$ is given by the following way: a homomorphism $R \rtimes F \rightarrow P$ is defined by $(r, f) \mapsto \mu \varphi(f)$ and an action of $P$ on $R \rtimes F$ by $^{p}(r, f)=\left({ }^{p} r,{ }^{p} f\right)$ for all $p \in P, r \in R, f \in F$.

One gets $R \rtimes F \underset{d_{1}}{\stackrel{d_{0}}{\Longrightarrow}} F, d_{0}(r, f)=f, d_{1}(r, f)=r f$.

It only remains and easy to prove that the homomorphism

$$
\alpha:\left(R /\langle\langle F, R\rangle\rangle_{(q)}\right) \times F /\langle F, F\rangle_{(q)} \longrightarrow T_{(q)}(R \rtimes F),
$$

defined by $\alpha([r],[f])=[(r, f)]$, is an isomorphism. 
Remark 2.5. For $\mu=0$ Theorem 2.4 generalizes the classical Hopf Formula from [BR] and for $q=0$ Proposition 2.3 and Theorem 2.4 show that one can get the first and the second homology of precrossed P-modules of Conduché and Ellis [CE] as nonabelian derived functors of the Peiffer abelianization functor.

Conjecture. Let $P$ be a group, $(M, \mu)$ be a precrossed $P$-module and $q$ be a nonnegative integer. Choose a precrossed $P$-module $(F, \partial)$ and $R_{1}, \ldots, R_{n}<_{P} F$ such that: $F / \prod_{1 \leqslant i \leqslant n} R_{i} \approx$ $M, H_{2}(F, q)_{P}=1$ and $H_{r}\left(F / \prod_{i \in A} R_{i}, q\right)_{P}=1$ for every proper subset $A \neq \emptyset$ of $\langle n\rangle=$ $\{1, \ldots, n\}, r=|A|+1$ and $|A|+2$ (for example, the precrossed $P$-modules $F / \prod_{i \in A} R_{i}$ are free for $A \neq\langle n\rangle)$. Then there is an isomorphism

$$
H_{n+1}(M, q)_{P} \approx\left\{\cap_{i=1}^{n} R_{i} \cap\langle F, F\rangle_{(q)}\right\} /\left\{\prod_{A \subseteq\langle n\rangle}\left\langle\left\langle\cap_{i \in A} R_{i}, \cap_{i \notin A} R_{i}\right\rangle\right\rangle_{(q)}\right\}
$$

\section{Some other results}

In this section we investigate low dimensional, first and second, homologies modulo $q$ of precrossed $P$-modules, always have in mind Proposition 2.3 and Theorem 2.4 and give some results generalizing in $q$ modular aspect the results of Conduché and Ellis [CE].

Proposition 3.1. Let $P$ be a group, $q$ be a nonnegative integer and

$$
1 \rightarrow L \rightarrow M \rightarrow N \rightarrow 1
$$

a short exact sequence of precrossed P-modules. Then there is an exact sequence of groups

$$
\begin{gathered}
H_{2}(M, q)_{P} \rightarrow H_{2}(N, q)_{P} \rightarrow L /\langle\langle M, L\rangle\rangle_{(q)} \\
\quad \rightarrow H_{1}(M, q)_{P} \rightarrow H_{1}(N, q)_{P} \rightarrow 1
\end{gathered}
$$

Proof. Suppose $1 \rightarrow R \rightarrow F \rightarrow M \rightarrow 1$ is a free presentation of the precrossed $P$-module $M$, and hence $1 \rightarrow R^{\prime} \rightarrow F \rightarrow N \rightarrow 1$ is a free presentation of the precrossed $P$-module $N$. Therefore $R \subset R^{\prime}$ implying $R \cap\langle F, F\rangle_{(q)} \subset R^{\prime} \cap\langle F, F\rangle_{(q)},\langle\langle F, R\rangle\rangle_{(q)} \subset\left\langle\left\langle F, R^{\prime}\right\rangle\right\rangle_{(q)}$ and there is the canonical group homomorphism $H_{2}(M, q)_{P} \rightarrow H_{2}(N, q)_{P}$.

The following commutative diagram of groups with exact rows

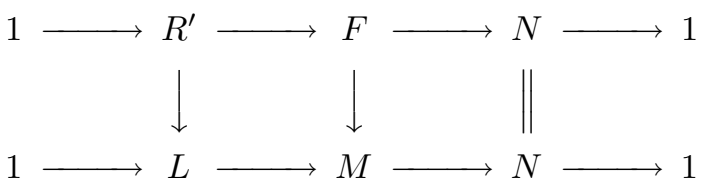

induces a homomorphism $H_{2}(N, q)_{P} \rightarrow L /\langle\langle M, L\rangle\rangle_{(q)}$.

Other homomorphisms are defined naturally and it is easy to check that the sequence (8) is exact.

Remark 3.2. One can extend the sequence (8) to any dimensions using the long exact sequence of the nonabelian derived functors and recovering for $\mu=0$ the eight term exact homology sequence of groups with coefficients in $\mathbb{Z}_{q}[E R]$.

The following result generalises the classical group result and uses the standard proof, originally due to $[\mathrm{S}]$.

For any precrossed $P$-module $(M, \mu)$ and any nonnegative integer $q$ there is the following family of precrossed $P$-submodules

$$
M_{(q)}^{(1)}=M, M_{(q)}^{(2)}=\langle\langle M, M\rangle\rangle_{(q)}, \ldots, M_{(q)}^{(n+1)}=\left\langle\left\langle M, M_{(q)}^{(n)}\right\rangle\right\rangle_{(q)} .
$$

Theorem 3.3. Let $P$ be a group, $q$ be a nonnegative integer and $\varphi: M \rightarrow N$ be a morphism of precrossed $P$-modules such that the following properties hold: 
(i) the natural homomorphism $H_{1}(M, q)_{P} \rightarrow H_{1}(N, q)_{P}$, induced by $\varphi$, is an isomorphism;

(ii) the natural homomorphism $\mathrm{H}_{2}(M, q)_{P} \rightarrow H_{2}(N, q)_{P}$, induced by $\varphi$, is a surjection.

Then $\varphi$ induces a natural isomorphism of precrossed $P$-modules

$$
M / M_{(q)}^{(n)} \stackrel{\approx}{\longrightarrow} N / N_{(q)}^{(n)} \quad \text { for } n \geqslant 2 .
$$

Proof. By induction. For $n=2$ the theorem is true. Suppose it is true for $n$. By Proposition 3.1 and the following commutative diagram of groups with exact rows

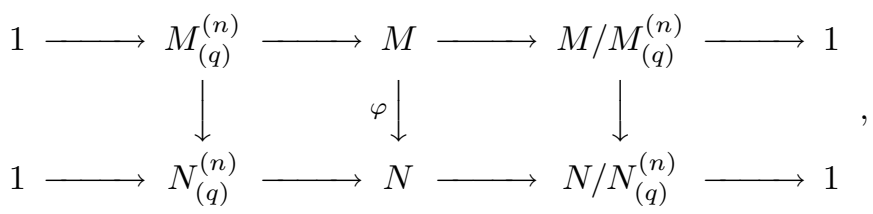

one has the following commutative diagram of groups with exact rows

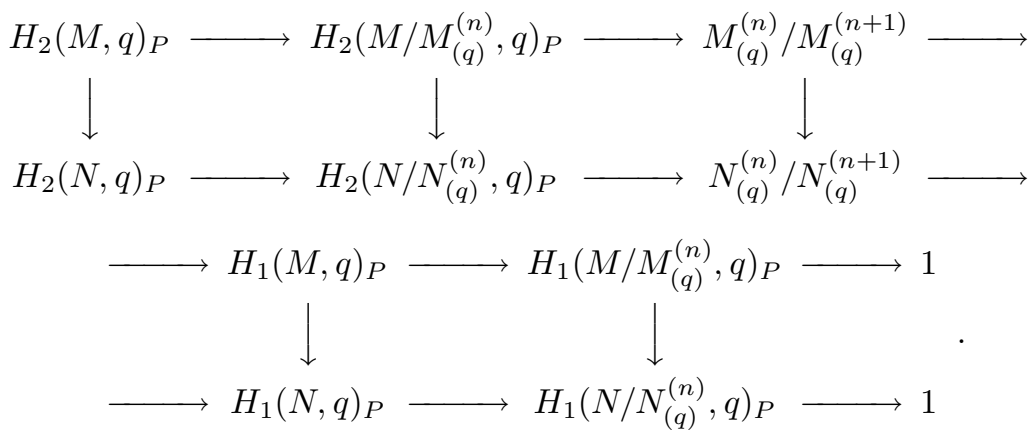

Using the "five lemma" $M_{(q)}^{(n)} / M_{(q)}^{(n+1)}$ is isomorphic to $N_{(q)}^{(n)} / N_{(q)}^{(n+1)}$. Then the following commutative diagram of groups

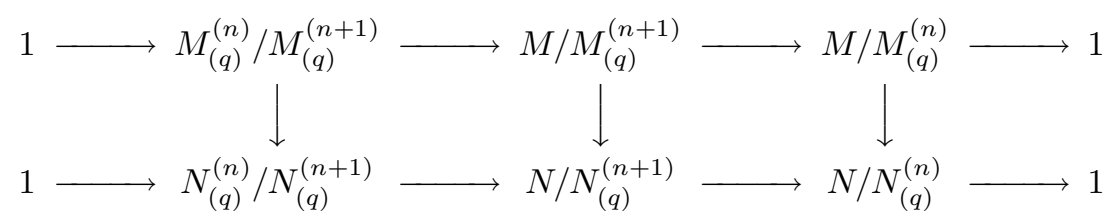

gives the result for $n+1$.

For any precrossed $P$-module $\mu: M \rightarrow P$, let $M \wedge_{P}^{q} M$ be the group generated by the symbols $m \wedge m^{\prime}$ and $\{k\}, m, m^{\prime} \in M, k \in \operatorname{Ker} \mu$ subject to the following relations:

$$
\begin{array}{r}
m \wedge m^{\prime} m^{\prime \prime}=\left(m \wedge m^{\prime}\right)\left(m \wedge m^{\prime \prime}\right)\left(\left\langle m, m^{\prime \prime}\right\rangle^{-1} \wedge{ }^{\mu m} m^{\prime}\right), \\
m m^{\prime} \wedge m^{\prime \prime}=\left(m \wedge m^{\prime} m^{\prime \prime} m^{\prime-1}\right)\left(\mu m^{\prime} m^{\prime} \wedge m^{\prime \prime}\right), \\
\left\langle m, m^{\prime}\right\rangle \wedge\left\langle n, n^{\prime}\right\rangle=\left(m \wedge m^{\prime}\right)\left(n \wedge n^{\prime}\right)\left(m \wedge m^{\prime}\right)^{-1}\left(n \wedge n^{\prime}\right)^{-1}, \\
\left(\left\langle m, m^{\prime}\right\rangle \wedge m^{\prime \prime}\right)\left(m^{\prime \prime} \wedge\left\langle m, m^{\prime}\right\rangle\right)=\left(m \wedge m^{\prime}\right)\left({ }^{\mu m^{\prime \prime}} m \wedge^{\mu m^{\prime \prime}} m^{\prime}\right)^{-1}, \\
k \wedge k=1, \\
\{k\}\left(m \wedge m^{\prime}\right)\{k\}^{-1}=\left(k^{q} m \wedge m^{\prime}\right)\left(k^{q} \wedge^{\mu m} m^{\prime}\right)^{-1}, \\
\left\{k k^{\prime}\right\}=\{k\} \prod_{i=1}^{q-1}\left(k^{-1} \wedge k^{1-q+i}\left(k^{\prime}\right)^{i} k^{q-1-i}\right)\left\{k^{\prime}\right\}, \\
\{k\}\left\{k^{\prime}\right\}\{k\}^{-1}\left\{k^{\prime}\right\}^{-1}=k^{q} \wedge k^{\prime q}, \\
\left\{\left\langle m, m^{\prime}\right\rangle\right\}=\left(m \wedge m^{\prime}\right)^{q}
\end{array}
$$

for all $m, m^{\prime}, m^{\prime \prime}, n, n^{\prime} \in M$ and $k, k^{\prime} \in \operatorname{Ker} \mu$. 
Note that (9)-(13) are the defining relations for the group $M \wedge_{P} M$ defined in [CE]. Furthermore, when $P=1$ or $\mu=0$ the group $M \wedge_{P}^{q} M$ coincides with the nonabelian exterior product modulo $q, M \wedge^{q} M$, introduced by Conduché and Rodriguez-Fernandez [CR] (see also $[\mathrm{B}],[\mathrm{ER}],[\mathrm{E}],[\mathrm{IN}])$.

There is an action of the group $P$ on the group $M \wedge_{P}^{q} M$ given by ${ }^{p}\left(m \wedge m^{\prime}\right)={ }^{p} m \wedge{ }^{p} m^{\prime}$ and ${ }^{p}\{k\}=\left\{{ }^{p} k\right\}$ for all $m, m^{\prime} \in M, k \in \operatorname{Ker} \mu$. Moreover, there exists a $P$-equivariant group homomorphism $\partial_{2}^{q}: M \wedge_{P}^{q} M \rightarrow M$ defined by $\partial_{2}^{q}\left(m \wedge m^{\prime}\right)=\left\langle m, m^{\prime}\right\rangle$ and $\partial_{2}^{q}(\{k\})=k^{q}$. It is clear that $\partial_{2}^{q}\left(M \wedge_{M}^{q} M\right)=M_{(q)}^{(2)}$.

Note that the complex of groups $M \wedge_{P}^{q} M \stackrel{\partial_{2}^{q}}{\longrightarrow} M \stackrel{\mu}{\longrightarrow} P$ is a 2-crossed module in the sence of Conduché $[\mathrm{C}]$.

Proposition 3.4. Let $(M, \mu)$ be a precrossed $P$-module, $q>0$ and

$$
1 \longrightarrow R \longrightarrow F \stackrel{\varphi}{\longrightarrow} M \longrightarrow 1
$$

a short exact sequence of precrossed $P$-modules, where $(F, \nu)$ is a free precrossed $P$-module. If the homomorphism $\partial_{2}^{q}: F \wedge_{P}^{q} F \rightarrow F$ is injective then the group $M \wedge_{P}^{q} M$ is isomorphic to the group $F_{(q)}^{(2)} /\langle\langle F, R\rangle\rangle_{(q)}$.

Proof. Let $L_{F}$ (resp. $\left.L_{M}\right)$ be the free group generated by the set $(F \times F) \cup \operatorname{Ker\nu }$ (resp. $(M \times M) \cup \operatorname{Ker} \mu)$. There is a commutative diagram of groups

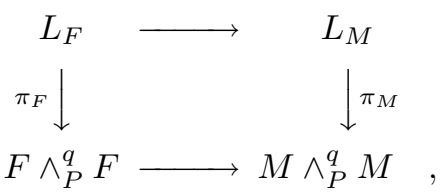

where the horizontal homomorphisms are surjective and $\pi_{F}$ and $\pi_{M}$ are canonical homomorphisms defined by $\pi_{F}\left(f, f^{\prime}\right)=f \wedge f^{\prime}, \pi_{F}(g)=\{g\}$ and $\pi_{F}\left(m, m^{\prime}\right)=m \wedge m^{\prime}, \pi_{F}(k)=\{k\}$ for all $f, f^{\prime} \in F, g \in \operatorname{Ker} \nu, m, m^{\prime} \in M$ and $k \in \operatorname{Ker} \mu$. It is easy to get that $\operatorname{Ker}\left(F \wedge_{P}^{q} F \rightarrow\right.$ $\left.M \wedge_{P}^{q} M\right)$ is the homomorphic image of $\operatorname{Ker}\left(L_{F} \rightarrow L_{M}\right)$ by $\pi_{F}$. It is also easy to check that $\operatorname{Ker}\left(L_{F} \rightarrow L_{M}\right)$ is the normal subgroup of $L_{F}$ generated by the elements $\left(f_{1}, f_{2}\right)\left(f^{\prime}{ }_{1}, f^{\prime}{ }_{2}\right)^{-1}$ and $f_{3} f_{3}^{\prime-1}$ such that $\varphi f_{i}=\varphi f^{\prime}{ }_{i}, f_{i}, f_{i}^{\prime} \in F(i=1,2)$ and $\varphi f_{3}=\varphi f^{\prime}{ }_{3}, f_{3}, f^{\prime}{ }_{3} \in \operatorname{Ker\nu }$. Thus its image in $F \wedge_{P}^{q} F$ is the normal subgroup generated by the elements $\left(f_{1} \wedge f_{2}\right)\left(f^{\prime}{ }_{1} \wedge f^{\prime}{ }_{2}\right)^{-1}$ and $\left\{f_{3}\right\}\left\{f^{\prime}{ }_{3}\right\}^{-1}$, which by the formulas (9), (10) and (16) coincides with the normal subgroup of $F \wedge_{P}^{q} F$ generated by the elements $f \wedge r, r \wedge f$ and $\{r\}, f \in F, r \in R$. Then the image of this subgroup by the isomorphism $F \wedge_{P}^{q} F \approx \partial_{2}^{q}\left(F \wedge_{P}^{q} F\right)=F_{(q)}^{(2)}$ is $\langle\langle F, R\rangle\rangle_{(q)}$ and thus $F_{(q)}^{(2)} /\langle\langle F, R\rangle\rangle_{(q)} \approx M \wedge_{P}^{q} M$.

Lemma 3.5. Let $h: A \rightarrow B$ and $g: B \rightarrow C$ be group homomorphisms. If $h$ is surjective then the following sequence of groups

$$
1 \longrightarrow \operatorname{Ker}(h) \longrightarrow \operatorname{Ker}(g h) \longrightarrow \operatorname{Ker}(g) \longrightarrow 1 \text {. }
$$

is exact.

Theorem 3.6. Let $\mu: M \rightarrow P$ be a precrossed $P$-module, $q>0$ and

$$
1 \longrightarrow R \longrightarrow F \stackrel{\varphi}{\longrightarrow} M \longrightarrow 1
$$

a short exact sequence of precrossed $P$-modules, where $F$ is a free precrossed $P$-module. If the homomorphism $\partial_{2}^{q}: F \wedge_{P}^{q} F \rightarrow F_{(q)}^{(2)}$ is an isomorphism then there is an isomorphism of groups

$$
H_{2}(M, q) \approx \operatorname{Ker}\left(M \wedge_{P}^{q} M \rightarrow M\right)
$$


Proof. By Lemma 3.5 one has the following exact sequence of groups

$$
1 \longrightarrow \operatorname{Ker}\left(\varphi \wedge_{P}^{q} \varphi\right) \longrightarrow \operatorname{Ker}\left(\partial_{2}^{q}\left(\varphi \wedge_{P}^{q} \varphi\right)\right) \longrightarrow \operatorname{Ker} \partial_{2}^{q} \longrightarrow 1
$$

From the commutative diagram of groups

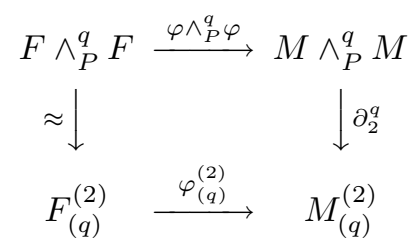

one gets $\operatorname{Ker}\left(\partial_{2}^{q}\left(\varphi \wedge_{P}^{q} \varphi\right)\right) \approx \operatorname{Ker} \varphi_{(q)}^{(2)}=R \cap F_{(q)}^{(2)}$. Then $\operatorname{Ker}\left(\partial_{2}^{q}: M \wedge_{P}^{q} M \rightarrow M\right) \approx$ $R \cap F_{(q)}^{(2)} /\langle\langle F, R\rangle\rangle_{(q)}=H_{2}(M, q)_{P}$

Finally we give an example showing that there exists such a group $P$ and a free precrossed $P$-module $F$ that the homomorphism $\partial_{2}^{q}: F \wedge_{P}^{q} F \rightarrow F$ is injective.

Lemma 3.7. Let $(M, \mu)$ be precrossed $P$-module and $q$ be a nonnegative integer. Then there is an exact sequence of groups

$$
M \wedge_{P} M \stackrel{\varphi}{\longrightarrow} M \wedge_{P}^{q} M \longrightarrow \operatorname{ker} \mu /\langle M, M\rangle \longrightarrow 1
$$

Proof. The homomorphism $\varphi$ is given by $\varphi\left(m \wedge m^{\prime}\right)=m \wedge m^{\prime}$. The required exacteness is easy to check.

Proposition-Example 3.8. Let $P$ be a free group, $\mu: F \rightarrow P$ be a free precrossed $P$-module and $q>0$. Then the homomorphism $\partial_{2}^{q}: F \wedge_{P}^{q} F \rightarrow F_{(q)}^{(2)}$ is an isomorphism.

Proof. Using Lemma 3.7 one has the following commutative diagram of groups with exact rows

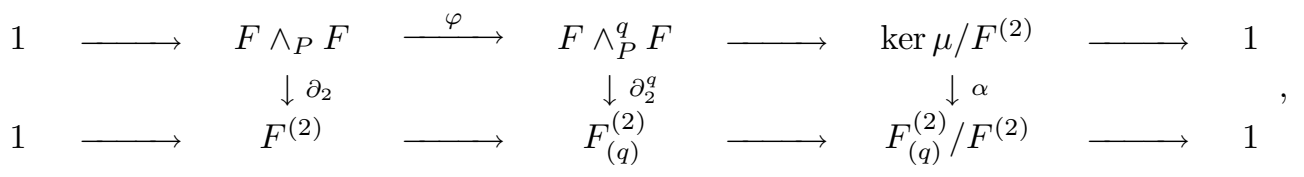

where $F^{(2)}=\langle F, F\rangle, \partial_{2}$ is an isomorphism [BC] proved applying a theorem of J.H.C. Whitehead [W] on 2-dimensional CW-complexes and the theorem of Kan [K] (see above) and hence $\varphi$ is injective. One can directly check that $\alpha$ is an isomorphism and so is $\partial_{2}^{q}$.

\section{References}

[B] R.Brown, q-perfect groups and q-central extensions, Publicaciones Mat. 34 (1990), 291-297.

[BB] M.Barr and J.Beck, "Homology and Standard Constructions", Lecture Notes in Mathematics, Vol.80, Springer-Verlag, Berlin/New York (1969), 245-335.

[BC] H.J.Baues and D.Conduché, The central series for Peiffer commutators in groups with operators, J.Algebra 133 (1) (1990), 1-34.

[BE] R.Brown and G.J.Ellis, Hopf formulae for the higher homology of a group, Bull. London Math. Soc. 20 (1988), 124-128.

[BH] R.Brown and J.Huebschmann, Identities among relations, dans "Low Dimensional Topology", L.M.S. Lecture Note 48, London, Cambrige Univ. Press, pp. 215-238, 1982. 
[BR] J.Barja and C.Rodriguez, Homology groups $H_{n}^{q}(-)$ and eight-term exact sequences, Cahiers Topologie Géom. Différentielle Catégoriques XXXI (1990), 91-120.

[CE] D.Conduché and G.J.Ellis Quelques propriétés homologiques des modules précroisés, J. Algebra 123 (2) (1989), 327-335.

[CR] D.Conduché and C.Rodriguez-Fernandez, Non-abelian tensor and exterior products modulo $q$ and universal $q$-central relative extension, J. Pure Appl. Algebra, 78 (1992), 139-160.

[CU1] E.B.Curtis, Lower central series of semi-simplicial complexes, Topology 2 (1963).

[CU2] E.B.Curtis, Some relations between homotopy and homology, Ann. of Math. 82 (1965), 386-413.

[E] G.J.Ellis, Tensor products and q-crossed modules, J. of London Math. Soc. (1995)

[ER] G.J.Ellis and C.Rodriguez, An exterior product for the homology of groups with integral coefficients modulo p, Cahiers Topologie Geom. Differentielle Categoriques XXX (1989), 339-344.

[IH] H.Inassaridze, Non-abelian homological algebra and its applications, Kluwer Academic Publishers, Amsterdam, 1997, 270 pages.

[IN] N.Inassaridze, Nonabelian tensor product modulo $q$ and $q$-homology of groups, (to appear).

[K] D.Kan, A relation between $\mathrm{CW}$-complexes and free c.s.s. groups, Amer. J. Math. 81 (1959), 512-528.

[P] T.Pirashvili, On non-abelian derived functors, Proc. A. Razmadze Math. Inst., Georgian Acad. Sci. 42 (1979), 91-104.

[Q] D.Quillen, Rational homotopy theory, Ann. of Math. 90 (1966), 205-295.

[S] J.R.Stallings, Homology and central series of groups, J. Algebra 2 (1965), 170-181.

[TV] M.Tierney and W.Vogel, Simplicial resolutions and derived functors, Math. Z. 111 (1969), 1-14.

[W] J.H.C.Whitehead, On adding relations to homotopy groups, Ann. of Math. 42 (1941), 409-428.

This article may be accessed via WWW at http://www.rmi.acnet.ge/hha/ or by anonymous ftp at ftp://ftp.rmi.acnet.ge/pub/hha/volumes/2000/n7/n7.(dvi,ps,dvi.gz,ps.gz)

Nick Inassaridze inas@rmi.acnet.ge

A. Razmadze Mathematical Institute

Georgian Academy of Sciences

1, M. Aleksidze St., Tbilisi 380093

Georgia

Emzar Khmaladze khmal@rmi.acnet.ge

A. Razmadze Mathematical Institute

Georgian Academy of Sciences

1, M. Aleksidze St., Tbilisi 380093

Georgia 